\title{
Deep Brain Stimulation of the Subthalamic Nucleus for Parkinson's Disease in a Patient with HIV Infection: Dual Clinical Benefit
}

\author{
Miguel F. Gago ${ }^{a, b} \quad$ Maria José Rosas ${ }^{a, b}$ Paulo Linhares ${ }^{b, d}$ \\ João Massano ${ }^{\text {b, d }}$ António Sarmento ${ }^{c, d}$ Rui Vaz ${ }^{b, d}$ \\ ${ }^{a}$ Department of Neurology, Centro Hospitalar do Alto Alve, EPE, Guimarães, \\ and ${ }^{\mathrm{b}}$ Movement Disorders and Functional Surgery Unit and ${ }^{\mathrm{C}}$ Department of \\ Infectology, Hospital de São João, EPE, and ${ }^{d}$ Faculdade de Medicina da \\ Universidade do Porto, Porto, Portugal
}

\section{Key Words}

Deep brain stimulation $\cdot$ Parkinson's disease $\cdot$ HIV $\cdot$ Hardware infection

\begin{abstract}
As a result of the efficacy of highly active antiretroviral therapy (HAART), patients with human immunodeficiency virus (HIV) can survive longer and are thus naturally prone to ageing-related degenerative disorders such as Parkinson's disease (PD). Managing PD and HIV in the same patient may be challenging, as HAART and levodopa interact and may cause intolerable side effects. Concerns about the increased risk of hardware infection in immunocompromised patients submitted to deep brain stimulation of the subthalamic nucleus (STN-DBS) still persist. We report a PD patient with HIV infection who suffered peakdose dyskinesias and intolerable gastrointestinal side effects while on HAART, prompting its suspension. STN-DBS allowed complete postoperative levodopa withdrawal and HAART restart, without infectious complications after 12 months of follow-up. STN-DBS seems to be a safe procedure in selected patients with both medically refractory PD and HIV infection, and may result in clinical optimization of both conditions.
\end{abstract}




\section{Introduction}

Modern highly active antiretroviral therapy (HAART) has brought considerable survival benefits for patients with human immunodeficiency virus (HIV) infection, a population now increasingly susceptible to the development of age-related neurodegenerative disorders $[1,2]$ such as Parkinson's disease (PD). Accordingly, patients who are diagnosed with HIV infection in the setting of previously diagnosed PD will have prolonged survival if treated with HAART, and will experience the full clinical course of PD. Amplified adverse effects of levodopa due to interactions with HAART, hindering optimal control of both conditions in the same patient, have been previously documented [3]. Deep brain stimulation of the subthalamic nucleus (STNDBS) is an effective treatment for medically refractory PD, while allowing levodopa therapy reduction [4-6]. However, concern remains regarding the theoretically increased risk of infection in immunocompromised patients following the implantation of external materials, such as DBS hardware. We describe a PD patient with concomitant HIV infection who underwent successful STN-DBS not complicated by infection, obtaining clinical benefits for both disorders.

\section{Case Report}

A 58-year-old Caucasian man was diagnosed with tremor-predominant PD at the age of 44 years. Dopamine agonists and levodopa therapy allowed a good symptomatic control. By the age of 48 years, he was diagnosed with HIV on a routine testing. Six years later, although he remained asymptomatic, the CD 4 count had reached 209 cells/ $\mu$ l, and HAART was started. Soon after, severe gastrointestinal symptoms (nausea, vomiting, and diarrhea) and peak-dose dyskinesias emerged, which were attributed to pharmacokinetic interactions between levodopa and HAART. Initially, levodopa was reduced at the cost of suboptimal control of PD, but afterwards HAART had to be discontinued because of intolerable dyskinesias. After 3 years of good symptomatic PD control and asymptomatic HIV infection, the patient began to suffer from severe motor fluctuations with morning off dystonia and peak-dose dyskinesias. By the time STN-DBS was considered, he was on immediate and controlled-release levodopa and ropinirole, totaling a daily levodopa equivalent dose of 1,250 mg. PD was in Hoehn-Yahr stage 3 while on medication, and the UPDRS-III score was 78 off medication and 18 after suprathreshold levodopa intake. There were no neurological, cognitive, or psychiatric disorders attributable to his HIV infection. With a preoperative CD4 level of 209 cells $/ \mu \mathrm{l}$ and a viral load of 348,395 copies/ml, the patient was placed on prophylactic co-trimoxazole before surgery. A preoperative cerebral MRI revealed slight periventricular white matter T2 hyperintensity. The usual surgical antibiotic prophylaxis (vancomycin plus cefotaxime) was used. The targeting and implantation of the leads in both STN were achieved by direct visualization on MRI and refined by intra-operative microelectrode recording and macrostimulation. The wires and the generator were inserted subcutaneously at the same time the leads were implanted, and no immediate postoperative adverse events were recorded. Early postoperative dyskinesias were observed. A cerebral CT scan excluded hemorrhages. Levodopa therapy was withdrawn, the patient was discharged without motor fluctuations with an UPDRS-III score of 11, and HAART (abacavir $300 \mathrm{mg}$ b.i.d., lamivudine $150 \mathrm{mg}$ i.d., darunavir $600 \mathrm{mg}$ b.i.d., and ritonavir $100 \mathrm{mg}$ b.i.d.) was restarted. Twelve months after surgery, he is without levodopa medication, has no motor fluctuations, and scores 21 in UPDRS-III, with the following stimulation settings: right electrode contact $0(2.8 \mathrm{~V} / 90 \mu \mathrm{s} / 210 \mathrm{~Hz})$ and left electrode contact $6(2.0 \mathrm{~V} / 90 \mu \mathrm{s} / 210 \mathrm{~Hz})$. He remains free of any surgery, stimulation, or medication-related adverse events, and presents a lower viral load $(1,053$ copies $/ \mathrm{ml}$ ) and an increased CD4 count (436 cells/ $\mu \mathrm{l})$. 


\section{Discussion}

The issue of medically refractory PD patients with concomitant HIV infection will likely become more important in the future as this population ages due to increased survival conferred by HAART. However, current evidence on this topic is scarce.

The incidence of movement disorders in HIV-infected patients is low (approximately 1\%) [7], and of these patients, about 50\% present with Parkinsonism related to the infection [8]. Also, some studies suggest that chronic HIV infection and long-term HAART may facilitate the development of PD via several mechanisms including inflammation, mitochondrial dysfunction, and interference with the ubiquitin proteasome pathway [2, 9]. In our patient the PD diagnosis preceded the diagnosis of HIV infection by 4 years. However, as PD, HIV infection is characterized by an insidious asymptomatic initial period. Although we can exclude the contribution of HAART, which was started after a PD course of several years and which was soon after suspended, we cannot determine the exact moment of HIV primo-infection and exclude its potential role in the neurodegenerative process of PD.

To the best of our knowledge, there is only one previous report of DBS for PD in a patient with coexistent HIV infection [10]. This patient was on HAART, was not immunocompromised (preoperative CD4 $=650$ cells $/ \mathrm{mm}^{3}$, undetectable circulating virus), and no postoperative infectious complications were reported; thus, DBS was suggested as a safe procedure in patients with normal CD4 levels. In contrast, our patient had much lower CD4 levels, and HAART could not be tolerated, presumably due to PD-HIV drug therapy interactions. The evidence presented here, albeit based on a single case report, is an important addition to that available so far, as several points are shown. First, a PD patient with concomitant HIV infection can obtain the same amount of motor benefit from STN-DBS as any other PD patient. Second, it is also possible to reduce and even cease the dopaminergic medication after STN-DBS. Finally, clinical benefits regarding HIV infection management may be gained from STN-DBS because levodopa may be suspended and HAART can successfully be introduced without the unbearable adverse effects related to their interaction.

\section{Acknowledgement}

We thank Dr. Rosa Sá, medical specialist of infectiology in the Hospital Universitário de Coimbra, for support in the clinical orientation of our patient. 


\section{References}

1 Broder S: The development of antiretroviral therapy and its impact on the HIV-1/AIDS pandemic. Antiviral Res 2010;85:1-18.

2 Brew BJ, Crowe SM, Landay A, Cysique LA, Guillemin G: Neurodegeneration and ageing in the HAART era. J Neuroimmune Pharmacol 2009;4:163-174.

-3 Caparros-Lefebvre D, Lannuzel A, Tiberghien F, Strobel M: Protease inhibitors enhance levodopa effects in Parkinson's disease. Mov Disord 1999;14:535.

-4 Deuschl G, Schade-Brittinger C, Krack P, et al: A randomized trial of deep-brain stimulation for Parkinson's disease. N Engl J Med 2006;355:896-908.

5 Follett KA, Weaver FM, Stern M, et al: Pallidal versus subthalamic deep-brain stimulation for Parkinson's disease. N Engl J Med 2010;362:2077-2091.

-6 Piboolnurak P, Lang AE, Lozano AM, Miyasaki JM, Saint-Cyr JA, Poon YY, Hutchison WD, Dostrovsky JO Moro E: Levodopa response in long-term bilateral subthalamic stimulation for Parkinson's disease. Mov Disord 2007;22:990-997.

7 Mattos JP, Rosso AL, Correa RB, Novis SA: Movement disorders in 28 HIV-infected patients. Arq Neuropsiquiatr 2002;60:525-530.

8 Cardoso F: HIV-related movement disorders: epidemiology, pathogenesis and management. CNS Drugs 2002;16:663-668.

\$9 Tisch S, Brew B: Parkinsonism in HIV-infected patients on highly active antiretroviral therapy. Neurology 2009;73:401-403.

10 Hettige S, Samuel M, Clough C, Hulse N, Ashkan K: Deep brain stimulation for Parkinson's disease when HIV coexists. Mov Disord 2009;24:2169-2171. 